\title{
THE GEOMETRY OF CONVEX CURVES TENDING TO 1 IN THE UNIT DISC
}

\author{
U. V. SATYANARAYANA AND MAX L. WEISS
}

\begin{abstract}
Let $f$ be a real convex function defined near $0^{*}$, $f(0)=f^{\prime}(0)=0, f>0$ otherwise. The family of curves, $f(\theta)=$ $\gamma(1-r), 0<\gamma<\infty$, in the unit disc is investigated. The union of the $w^{*}$ closures in the maximal ideal space of $H^{\infty}$ of these curves is seen to be a union of nontrivial parts and each such part is hit by the closure of each such curve. Using results of Hoffman and the Wermer map onto such a part, nets in the disc tending to points of the part are located on the curves as explicitly as can be expected. One striking consequence of this coordinatization is the fact that the closure of any such curve in a part is invariably the image under the Wermer map of an oricycle. This is not true for the limiting case of the family of Stolz rays at 1 . Other detailed results of a similar nature are derived.
\end{abstract}

1. Introduction and preliminaries. Let $D$ be the open unit disc in the complex plane. We assume that the reader is somewhat familiar with the theory of $H^{\infty}(D)$ as a function algebra including such basic concepts as its maximal ideal space, the fiber $\mathscr{D}_{1}$ above 1 , and the results of Hoffman's work [2] on the parts of $H^{\infty}$.

Using results in $[2, \S 6]$, it is not difficult to see that two nontangential curves in $D$ tending to 1 have the same homomorphisms in $\mathscr{D}_{1}$ in their closure if and only if they are tangent to each other at 1 . Furthermore, the collection of all nontangential homomorphisms is a union of nontrivial parts each of which is hit by the closure of each Stolz ray. In contrast, any two curves tending to 1 tangent on the same side to the unit circle are, of course, tangent to each other but by no means need to share homomorphisms or even parts of $\mathscr{D}_{1}$ in their closures. One begins to see from [4] the wealth of possible behavior of $H^{\infty}$ functions along curves. It is shown there that if $\Gamma$ is any upper tangential curve at 1 there is a Blaschke product $B$, whose only singularity is at 1 , which tends to zero along $\Gamma$. Consequently, there is a curve to 1 more tangential than $\Gamma$ along which $B$ has cluster values of modulus 1 only.

Several kinds of questions arise naturally. How strong an "order of contact" do two upper tangential curves have to have at 1 for their closures

Received by the editors August 3, 1972 and, in revised form, February 5, 1973. AMS (MOS) subject classifications (1970). Primary 30A98.

Key words and phrases. Convex curve, Wermer map, part, $H^{\infty}$. 
to be identical in $\mathscr{D}_{1}$ ? How "far apart" do such curves have to be for the closures in $\mathscr{D}_{1}$ to be disjoint or at least different? Given an upper tangential curve $\Gamma$, what corresponding family consists of curves which represent exactly the same parts as $\Gamma$ by their closures in $\mathscr{D}_{1}$ ? What does the closure of a curve in a part look like? Some answers to these questions follow from results of [2] phrased in terms of the pseudohyperbolic metric, $\chi$.

THEOREM (HOFFMAN). The natural extension of $\chi$ to the maximal ideal space of $H^{\infty}$ is lower semicontinuous. If $S$ and $T$ are subsets of the disc $D$ and if a nontrivial (i.e., interpolating) homomorphism belongs to the closure of each set, then the pseudohyperbolic distance (at 1) from $S$ to $T$ is zero.

Thus, combining the lower semicontinuity of $\chi$ with Pick's theorem, we see that if two curves have (pseudohyperbolic) Hausdorff distance zero, then they have identical closures. The second statement of Hoffman's theorem immediately shows when curves have different closures. From this second statement it is shown in [3] that two sets represent the same parts in their closures if and only if their Hausdorff distance is less than 1. In this paper we will make these results explicit for the case of convex curves to 1 .

Before proceeding to the results of this paper we wish to indicate some of the subtleties involved. For example, it is not immediately clear how to measure the "order of contact" between two curves to obtain answers to the above questions. Because of the possibility of wild oscillations of some tangential curves we do not offer complete solutions, but restrict ourselves to the family of convex curves at 1 . Here even the choice of equation for such a curve is important. For example, let $f(\theta)=\exp \left(-0^{-1}\right)$ and consider the two upper tangential curves, $f(\theta)=(1-r)$ and $f(\theta)=2(1-r)$. We will see in $\S 2$ that these two curves have different closures in $\mathscr{D}_{1}$. However, if one measures the "order of contact" of these two curves by studying the limit of $\theta / f^{-1}(1-r)$ along the curves one easily computes that in both cases this ratio tends to 1. That is, in one description the two curves have the same "order of contact" but different closures while in the other description they have the desired different "order of contact." It can be further shown that the set of homomorphisms which are limits of nets $\left\{r_{\alpha} e^{i \theta_{\alpha}}\right\}$ of the single "order of tangency", $\theta_{\alpha} / f^{-1}\left(1-r_{\alpha}\right) \rightarrow 1$ is a union of parts. This gives a somewhat blurry contrast to the above-mentioned situation for nontangential curves.

Given a real-valued continuous function $f$ defined near 0 with $f(0)=0$, $f \neq 0$ otherwise, we say that a curve $\Gamma$ [a homomorphism $h$ ] has order $(f, \gamma)$ provided $f(\theta) /(1-r) \rightarrow \gamma$ as $r e^{i \theta} \rightarrow 1$ along $\mathrm{V}\left[f\left(\theta_{\alpha}\right) /\left(1-r_{\alpha}\right) \rightarrow \gamma\right.$ for some net $\left.r_{\alpha} e^{i \theta_{\alpha} \rightarrow h}\right],-\infty \leqq \gamma \leqq \infty$. For nontangential approaches the single 
function $f(\theta)=0$ suffices. In this paper we will be mainly concerned with upper tangential approaches. We then naturally have $f$ defined only near $0^{+}, f(0)=f^{\prime}(0)=0, f>0$ otherwise and $0 \leqq \gamma \leqq \infty$. Let $\mathscr{K}$ denote the family of all such functions for which $f$ is, in addition, convex near $0^{+}$. For $f \in \mathscr{K}$ and $0 \leqq \gamma \leqq \infty$, let

$$
A(f, \gamma)=\left\{h \in \mathscr{D}_{1}: h \text { has order }(f, \gamma)\right\}
$$

and for $0<\gamma<\infty$ let

$$
\Gamma(f, \gamma)=\left\{r e^{i \theta}: f(\theta)=\gamma(1-r)\right\} .
$$

Strictly speaking, the orders corresponding to $\gamma=0$ or $\infty$ are not really specific orders. For example, $A(f, 0)$ consists of all upper tangential homomorphisms which can be approached less tangentially than all homomorphisms in $A(f, \gamma), 0<\gamma<\infty$. Furthermore, there are homomorphisms in $\mathscr{D}_{1}$ which in this sense have no definite order. From [1] we see that the so-called upper barely tangential homomorphisms are in $A(f, \gamma)$ if and only if $\gamma=0$. Similarly, [4], if $h$ is in the upper boundary of $\mathscr{D}_{1}$ (relative to the union of the fibers above the unit circle), then $h \in$ $A(f, \gamma)$ if and only if $\gamma=\infty$.

2. The results. Given $z_{0}=r_{0} e^{i \theta_{0}}$ in $D$ let

$$
\begin{aligned}
& a\left(z_{0}\right)=\frac{1-r_{0}^{2}}{1+2 r_{0} \cos \theta_{0}+r_{0}^{2}}=\operatorname{Re}\left(\frac{1-z_{0}}{1+z_{0}}\right), \\
& b\left(z_{0}\right)=\frac{2 r_{0} \sin \theta_{0}}{1+2 r_{0} \cos \theta_{0}+r_{0}^{2}}=-\operatorname{Im}\left(\frac{1-z_{0}}{1+z_{0}}\right) .
\end{aligned}
$$

We first prove several technical lemmas.

Lemma 1. Let $z_{\alpha}=r_{\alpha} e^{i \theta_{\alpha}}$ be a net in $D$ tending to 1 . Let $z_{0} \in D$ and let $\rho_{\alpha} e^{i \varphi \alpha}=\left(z_{\alpha}+z_{0}\right) /\left(1+\bar{z}_{\alpha} z_{0}\right)$. Then,

$$
\left(1-\rho_{\alpha}\right) /\left(1-r_{\alpha}\right) \rightarrow a\left(z_{0}\right), \quad\left(\varphi_{\alpha}-0_{\alpha}\right) /\left(1-r_{\alpha}\right) \rightarrow b\left(z_{0}\right) .
$$

Proof. This is easily computed beginning with the fact that

$$
1-\rho e^{i(\varphi-\theta)}=(1-r)\left[\left(r-\bar{z} z_{0}\right) /\left(r\left(1+\bar{z} z_{0}\right)\right)\right] .
$$

Lemma 2. Let $f \in \mathscr{K}$. Then, for any fixed constant $k$,

$$
\lim _{\theta \rightarrow 0^{+}} \frac{f(\theta+k f(\theta))}{f(\theta)}=1 .
$$

Proof. Since $f$ is convex and $f(0)=0$, there is a nondecreasing integrable function $g$ such that, near $0^{+}, f(\theta)=\int_{0}^{\theta} g(x) d x$. Since $f^{\prime}(0)=0, g$ 
has essential limit zero at $0^{+}$. Then

Since

$$
\frac{f(\theta+k f(\theta))}{f(\theta)}=\frac{1}{f(\theta)} \int_{0}^{\theta+k f(\theta)} g(x) d x=1+\frac{1}{f(\theta)} \int_{\theta}^{\theta+k f(\theta)} g(x) d x .
$$

$\left|\frac{1}{f(\theta)} \int_{\theta}^{\theta+k i(\theta)} g(x) d x\right|$

the result follows.

$$
\begin{aligned}
& \leqq \frac{|k| f(\theta)[\operatorname{ess} \sup \{g(x): 0 \leqq x \leqq \max (\theta, \theta+k f(\theta))\}]}{f(\theta)} \\
& \rightarrow 0 \quad \text { as } \theta \rightarrow 0^{\prime}
\end{aligned}
$$

Lemma 3. Let $z_{\alpha}=r_{\alpha} e^{i \theta_{\alpha}}, w_{\alpha}=\rho_{\alpha} e^{i \varphi_{\alpha}}$ be two nets in $D$ tending to 1 (with the same directed set $)$. Then, the distances $\chi\left(z_{\alpha}, w_{\alpha}\right)$ tend to zero if and only if

$$
\left(1-r_{\alpha}\right) /\left(1-\rho_{\alpha}\right) \rightarrow 1 \text { and }\left(\rho_{\alpha}-\varphi_{\alpha}\right)^{2} /\left(1-r_{\alpha}\right)\left(1-\rho_{\alpha}\right) \rightarrow 0 \text {. }
$$

Proof. If one analyzes the identity

$$
1-\chi^{2}\left(z_{\alpha}, w_{\alpha}\right)=\frac{\left(1+r_{\alpha}\right)\left(1+\rho_{\alpha}\right)}{\frac{1-r_{\alpha}}{1-\rho_{\alpha}}+2 r_{\alpha}+r_{\alpha}^{2} \frac{1-\rho_{\alpha}}{1-r_{\alpha}}+2 r_{\alpha} \rho_{\alpha} \frac{1-\cos \left(\theta_{\alpha}-\varphi_{\alpha}\right)}{\left(1-r_{\alpha}\right)\left(1-\rho_{\alpha}\right)}}
$$

the lemma is clear.

The next theorem is one of our basic computations.

TheOREM 1. Let $f \in \mathscr{K}$. Let $z_{\alpha}=r_{\alpha} e^{i \theta_{\alpha}}$ be a net in D tending to 1 and ${ }^{*} *$ to the homomorphism $h \in \mathscr{D}_{1}$. Suppose $h$ has order $(f, \gamma), 0<\gamma<\infty$. If $\tau$ is the Wermer map constructed in [2] of $D$ onto the part of $h$ such that $\tau(0)=h$, then, for any point $z_{0}=r_{0} e^{i \theta_{0}}$ in $D, \tau\left(z_{0}\right)=\lim _{\alpha} \rho_{\alpha}^{\prime} e^{i \varphi_{\alpha^{\prime}}}$ where

$$
\varphi_{\alpha}^{\prime}=\theta_{\alpha}+b\left(z_{0}\right) f\left(\theta_{\alpha}\right) / \gamma, \quad \rho_{\alpha}^{\prime}=1-f\left(\varphi_{\alpha}^{\prime}\right) a\left(z_{0}\right) / \gamma .
$$

Proof. By a basic result of Hoffman [2], $\tau\left(z_{0}\right)=\lim _{\alpha} \rho_{\alpha} e^{i \varphi_{\alpha}}$ where

$$
\rho_{\alpha} e^{i \varphi_{\alpha}}=\left(z_{\alpha}+z_{0}\right) /\left(1+\bar{z}_{\alpha} z_{0}\right) \text {. }
$$

Thus, since $\chi$ is lower semicontinuous it is sufficient to show that $\lim \chi\left(\rho_{\alpha} e^{i \varphi_{\alpha}}, \rho_{\alpha}^{\prime} e^{i \varphi_{\alpha^{\prime}}}\right)=0$. First,

$$
\frac{1-\rho_{\alpha}}{1-\rho_{\alpha}^{\prime}}=\frac{1}{a\left(z_{0}\right)} \frac{1-\rho_{\alpha}}{1-r_{\alpha}}\left[\frac{1}{\gamma} \frac{f\left(\theta_{\alpha}\right)}{1-r_{\alpha}} \frac{f\left(\varphi_{\alpha}^{\prime}\right)}{f\left(\theta_{\alpha}\right)}\right]^{-1} .
$$

Using the fact that $h$ has order $(f, \gamma)$, the definition of $\varphi_{\alpha}^{\prime}$, and Lemmas 1 and 2 it is clear that $\left(1-\rho_{\alpha}\right) /\left(1-\rho_{\alpha}^{\prime}\right) \rightarrow 1$. Second,

$$
\frac{\left(\varphi_{\alpha}-\varphi_{\alpha}^{\prime}\right)^{2}}{\left(1-\rho_{\alpha}\right)\left(1-\rho_{\alpha}^{\prime}\right)}=\left(\frac{1-r_{\alpha}}{1-\rho_{\alpha}}\right)^{2}\left(\frac{1-\rho_{\alpha}}{1-\rho_{\alpha}^{\prime}}\right)\left[\frac{\varphi_{\alpha}-\theta_{\alpha}}{1-r_{\alpha}}-b\left(z_{0}\right) \frac{1}{\gamma} \frac{f\left(\theta_{\alpha}\right)}{1-r_{\alpha}}\right]^{2} \text {. }
$$


Again, using Lemmas 1 and 2 and the result we just proved we see that $\left(\varphi_{\alpha}-\varphi_{\alpha}^{\prime}\right)^{2} /\left(1-\rho_{\alpha}\right)\left(1-\rho_{\alpha}^{\prime}\right) \rightarrow 0$. Therefore, $\chi\left(\rho_{\alpha} e^{i \varphi_{\alpha}}, \rho_{\alpha}^{\prime} e^{i \varphi_{\alpha}^{\prime}}\right) \rightarrow 0$ by Lemma 3 .

We can see quickly from Theorem 1 that the approaches $r e^{i \theta} \rightarrow 1$ "asymptotic" to the curve $\Gamma(f, \gamma)$ in the sense that $f(0) /(1-r) \rightarrow \gamma$ give no new homomorphisms. This is the content of the next theorem.

TheOREM 2. For $f \in \mathscr{K}$ and $0<\gamma<\infty, A(f, \gamma)=\Gamma(f, \gamma)-\Gamma(f, \gamma)$ where the bar denotes $w^{*}$ closure.

ProOF. It is clear that the right-hand side of the equality is a subset of the left. On the other hand, suppose $h \in A(f, \gamma), h=\lim r_{\alpha} e^{i \theta_{\alpha}}$ with

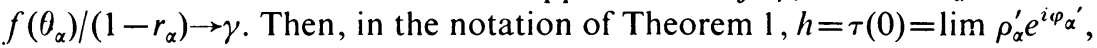
where for $z_{0}=0$ we have $a\left(z_{0}\right)=1, b\left(z_{0}\right)=0$ and $\varphi_{\alpha}^{\prime}=0_{\alpha}, \rho_{\alpha}^{\prime}=1-\gamma^{-1} f\left(\varphi_{\alpha}^{\prime}\right)$. Clearly, $f\left(\varphi_{\alpha}^{\prime}\right) /\left(1-\rho_{\alpha}^{\prime}\right)=\gamma$ so $h \in \Gamma(f, \gamma)^{-}-\Gamma(f, \gamma)$ and the equality follows.

We next give an answer to the question about the difference of the closures in $\mathscr{D}_{1}$ of two distinct curves $\Gamma(f, \gamma)$ and $\Gamma\left(f, \gamma^{\prime}\right)$. This answer is partly based on a result of Wortman [5, Theorem 3.6] from which it immediately follows that every homomorphism in $A(f, \gamma)$ is nontrivial.

THEOREM 3. Let $f \in \mathscr{K}, \gamma \neq \gamma^{\prime}$ in $(0, \infty)$. Then, the set $A(f, \gamma) \cap$ $A\left(f, \gamma^{\prime}\right)=\varnothing$.

Proof. Suppose $h \in A(f, \gamma) \cap A\left(f, \gamma^{\prime}\right)$. Then $h$ is a nontrivial homomorphism. Using Theorem 2 and the second statement of Hoffman's theorem in $\S 1$, there are nets $r_{\alpha} e^{i \theta_{\alpha}}$ on $\Gamma(f, \gamma)$ and $\rho_{\alpha} e^{i \varphi_{\alpha}}$ on $\Gamma\left(f, \gamma^{\prime}\right)$ tending to $h$ with $\chi\left(r_{\alpha} e^{i \theta_{\alpha}}, \rho_{\alpha} e^{i \varphi_{\alpha}}\right) \rightarrow 0$. By Lemma 3 ,

$$
\frac{1-r_{\alpha}}{1-\rho_{\alpha}} \rightarrow 1 \text { and } \frac{\left(\theta_{\alpha}-\varphi_{\alpha}\right)^{2}}{\left(1-r_{\alpha}\right)\left(1-\rho_{\alpha}\right)} \rightarrow 0 \text {. }
$$

But we may write (omitting subscripts)

$$
\frac{(\theta-\varphi)^{2}}{(1-r)(1-\rho)}=\left[\frac{f(\varphi)}{1-\rho}\right]^{2}\left(\frac{1-\rho}{1-r}\right)\left[\frac{1-f(\theta) / f(\varphi)}{(f(\varphi)-f(\theta)) /(\varphi-\theta)}\right]^{2} .
$$

The first factor on the right equals $\gamma^{\prime 2}$ and the second tends to 1 . The difference quotient in the third factor is bounded since $f \in \mathscr{K}$. Keeping in mind that the left-hand side tends to 0 , we see that $f(\theta) / f(\varphi) \rightarrow 1$. However, $f(0) / f(\varphi)=\gamma(1-r) / \gamma^{\prime}(1-\rho) \rightarrow \gamma / \gamma^{\prime}$ and we conclude that $\gamma=\gamma^{\prime}$.

We are now in a position to discuss rather fully the implications of the "coordinatizations" of the various parts given in Theorem 1. In the same spirit as the above calculations it is straightforward to compute the corresponding results for the Stolz curves. Let $r_{\alpha} e^{i \theta_{\alpha \rightarrow h}}$ in such a way that $\theta_{\alpha} /\left(1-r_{\alpha}\right) \rightarrow \gamma \in(-\infty, \infty)$. Let $\tau$ be the Wermer map onto the part of $h$ 
such that $\tau(0)=h$. Then, for any $z_{0} \in D$

$$
\begin{aligned}
\tau\left(z_{0}\right) & =\lim \begin{cases}\left(1-a\left(z_{0}\right) \theta_{\alpha} / \gamma\right) e^{i\left(\theta+\left(b\left(z_{0}\right) / \gamma\right) \theta\right)}, & \gamma \neq 0, \\
\left(1-a\left(z_{0}\right)\left(1-r_{\alpha}\right)\right) e^{i b\left(z_{0}\right)\left(1-r_{\alpha}\right)}, & \gamma=0,\end{cases} \\
& =\lim \left(1-a\left(z_{0}\right)\left(1-r_{\alpha}\right)\right) e^{i\left(\gamma+b\left(z_{0}\right)\right)\left(1-r_{\alpha}\right) .}
\end{aligned}
$$

Consequently, the closure of the nontangential curve $0=\gamma^{\prime}(1-r)$ in the part is the image under $\tau$ of the circle

$$
\begin{array}{rlrl}
\left(x+\frac{\gamma}{\gamma+\gamma^{\prime}}\right)^{2}+\left(y+\frac{1}{\gamma+\gamma^{\prime}}\right)^{2} & =\frac{1+\gamma^{\prime 2}}{\left(\gamma+\gamma^{\prime}\right)^{2}}, & \gamma^{\prime} & \neq-\gamma, \\
\gamma x+y & =-\gamma, & \gamma^{\prime}=-\gamma,
\end{array}
$$

which is one of the circles in the family of Steiner circles with centers on $x=\gamma y$ and passing through $(-1,0)$ and $\left(\left(1-\gamma^{2}\right)\left(1+\gamma^{2}\right)^{-1},-2 \gamma\left(1+\gamma^{2}\right)^{-1}\right)$. Also $\gamma^{\prime}=\left(\gamma+b\left(z_{0}\right)\right) / a\left(z_{0}\right)$.

One would expect the situation to be at least as complicated for the family of curves $\Gamma(f, \gamma)$. However, as we shall see, the much studied nontangential approaches rather than being the model for generalization are in this case an anomaly.

We observe that for $d>0$, the level curve $a(z)=d$ is the oricycle, $C_{d}$, internally tangent to the unit circle at -1 whose cartesian equation is

$$
(x+d /(1+d))^{2}+y^{2}=(1 /(1+d))^{2} .
$$

From Theorem 1 we see that given $z_{0} \in D, \tau\left(z_{0}\right) \in A\left(f, \gamma / a\left(z_{0}\right)\right)$ since $f\left(\varphi_{\alpha}^{\prime}\right) /\left(1-\rho_{\alpha}^{\prime}\right)=\gamma / a\left(z_{0}\right)$. Thus, the image of the oricycle, $C_{a\left(z_{0}\right)}$, is contained in $A\left(f, \gamma / a\left(z_{0}\right)\right)$. On the other hand if $\tau\left(z_{0}\right)$ is nontrivial, and $\tau\left(z_{0}\right) \in A\left(f, \gamma^{\prime}\right)$, we see from Theorem 3 that $\gamma^{\prime}=\gamma / a\left(z_{0}\right)$ and thus the image of $C_{a\left(z_{0}\right)}$ contains $A\left(f, \gamma / a\left(z_{0}\right)\right)$. The following corollaries should by now be evident.

TheOREM 4. Let $f \in \mathscr{K}$ and let $A(f)=\bigcup\{A(f, \gamma): 0<\gamma<\infty\}$. Then,

(1) $A(f)$ is an open union of nontrivial parts.

(2) Each part in $A(f)$ intersects the $w^{*}$ closure of each curve $\Gamma(f, \gamma)$, $0<\gamma<\infty$.

(3) For each fixed $\gamma \in(0, \infty), h \in A(f, \gamma)$, let $\tau$ be the Wermer map of $D$ onto the part, $P=P(h)$, of $h$ such that $\tau(0)=h$. Then, for each $\gamma^{\prime} \in(0, \infty)$, $A\left(f, \gamma^{\prime}\right) \cap P=\tau\left(C_{y / \gamma^{\prime}}\right)$.

Consequently, the $w^{* *}$ closure of each curve, $\Gamma(f, \gamma), 0<\gamma<\infty$, intersects each part of $A(f)$ in the image under a Wermer map of an oricycle.

The level curve $b(z)=d,-\infty<d<\infty$, is the circle, $H_{t}$, tangent from within $D$ at -1 to the real diameter whose equation is

$$
(x+1)^{2}+\left(y-d^{-1}\right)^{2}=d^{-2} .
$$


With the notation of Theorem 1 , each $z_{0} \in D$ lies on the intersection of unique circles $C_{a\left(z_{0}\right)}, H_{b\left(z_{0}\right)}$. The net $\rho_{\alpha}^{\prime} e^{i \varphi_{\alpha}^{\prime}}$ tending to $\tau\left(z_{0}\right)$ is obtained by advancing or retarding the original angle $\theta_{\alpha}$ to $\theta_{\alpha}+b\left(z_{0}\right) f\left(\theta_{\alpha}\right) \gamma^{-1}$ and then choosing $\rho_{\alpha}^{\prime}$ so that $\rho_{\alpha} e^{i \varphi_{\alpha^{\prime}}} \in \Gamma\left(f, \gamma / a\left(z_{0}\right)\right)$. In particular, the real diameter of $D$ corresponds to $b(z)=0$. Thus, the closure of each curve $\Gamma\left(f, \gamma^{\prime}\right)$ intersects the $\tau$-image of the diameter in simply

$$
h^{\prime}=\lim \left(1-f\left(\theta_{\alpha}\right) a\left(z_{0}\right) \gamma^{-1}\right) e^{i \theta_{\alpha}} .
$$

It is possible now to return to the original technique involving the pseudo-hyperbolic metric and compute the Hausdorff distance between two curves $\Gamma(f, \gamma)$ and $\Gamma\left(f, \gamma^{\prime}\right)$.

THEOREM 5. Let $f \in \mathscr{K}, 0<\gamma<\infty$. Given a homomorphism $h \in A(f, \gamma)$, for any $0<\gamma^{\prime}<\infty$ and any $h^{\prime} \in A\left(f, \gamma^{\prime}\right)$

$$
\chi\left(h, h^{\prime}\right) \geqq\left|\left(\gamma-\gamma^{\prime}\right) /\left(\gamma+\gamma^{\prime}\right)\right|
$$

and there is exactly one such $h^{\prime}$ for which equality holds.

Consequently, the (pseudo-hyperbolic) Hausdorff distance (at 1) between $\Gamma(f, \gamma)$ and $\Gamma\left(f, \gamma^{\prime}\right)$ is exactly $\left|\gamma-\gamma^{\prime}\right| /\left|\gamma+\gamma^{\prime}\right|$.

Proof. If $h^{\prime}$ is in a different part from $h$, the inequality certainly holds. Otherwise, let $\tau$ be the Wermer map of $D$ onto the part, $P$, of $h$ with $\tau(0)=h$. Let $h^{\prime}=\tau\left(z_{0}\right)$. In [2] Hoffman proved that in case $P$ is a nontrivial part, $\tau$ is an isometry. Thus, $\chi\left(h, h^{\prime}\right)=\chi\left(\tau(0), \tau\left(z_{0}\right)\right)=\chi\left(0, z_{0}\right)=r_{0}$. Since $\gamma^{\prime}=\gamma / a\left(z_{0}\right)$ we have

$$
\left|\frac{\gamma-\gamma^{\prime}}{\gamma+\gamma^{\prime}}\right|=\left|\frac{a\left(z_{0}\right)-1}{a\left(z_{0}\right)+1}\right|=r_{0}\left|\frac{r_{0}+\cos \theta_{0}}{1+r_{0} \cos \theta_{0}}\right| \leqq r_{0}=\chi\left(h, h^{\prime}\right),
$$

and equality occurs if and only if $z_{0}=\left(\gamma-\gamma^{\prime}\right) /\left(\gamma+\gamma^{\prime}\right)\left(= \pm r_{0}\right)$ since the oricycle $C_{\gamma / \gamma^{\prime}}$ intersects the real diameter only at that point.

To verify the last assertion we show that the $\varepsilon$ pseudo-hyperbolic sheath about $\Gamma\left(f, \gamma^{\prime}\right)$ contains a terminal arc of $\Gamma(f, \gamma)$ if $\varepsilon>$ $\left|\gamma-\gamma^{\prime}\right| /\left|\gamma+\gamma^{\prime}\right|$ and omits a terminal arc if $\varepsilon<\left|\gamma-\gamma^{\prime}\right| /\left|\gamma+\gamma^{\prime}\right|$. The result then follows upon interchanging the roles of $\gamma$ and $\gamma^{\prime}$.

Suppose $\varepsilon$ satisfies the first above-mentioned inequality but the $\varepsilon$ sheath about $\Gamma\left(f, \gamma^{\prime}\right)$ fails to contain a terminal arc of $\Gamma(f, \gamma)$. Recall that every sequence tending to 1 has an interpolating subsequence. Thus, we may find a net $r_{\alpha} e^{i \theta_{\alpha}} \in \Gamma(f, \gamma)$ which is omitted by the $\varepsilon$ sheath about $\Gamma\left(f, \gamma^{\prime}\right)$ which tends to a nontrivial homomorphism $h$. In the notation of 
Theorem 1 choose $z_{0}=\left(\gamma-\gamma^{\prime}\right) /\left(\gamma+\gamma^{\prime}\right)$ and $h^{\prime}=\tau\left(z_{0}\right)$. Then, $\rho_{\alpha}^{\prime} e^{i \varphi_{\alpha}^{\prime}} \in$ $\Gamma\left(f, \gamma^{\prime}\right)$ so using the first part of this theorem

$$
\begin{aligned}
\varepsilon & \leqq \chi\left(r_{\alpha} e^{i \theta_{\alpha}}, \rho_{\alpha}^{\prime} e^{i \varphi_{\alpha}^{\prime}}\right) \leqq \chi\left(r_{\alpha} e^{i \theta_{\alpha}}, \rho_{\alpha} e^{i \varphi_{\alpha}}\right)+\chi\left(\rho_{\alpha} e^{i \varphi_{\alpha}}, \rho_{\alpha}^{\prime} e^{i \varphi_{\alpha}^{\prime}}\right) \\
& =\left|\frac{\gamma-\gamma^{\prime}}{\gamma+\gamma^{\prime}}\right|+\chi\left(\rho_{\alpha} e^{i \varphi_{\alpha}}, \rho_{\alpha}^{\prime} e^{i \varphi_{\alpha}^{\prime}}\right) .
\end{aligned}
$$

Taking a limit we obtain $\varepsilon \leqq\left|\gamma-\gamma^{\prime}\right| /\left|\gamma+\gamma^{\prime}\right|$ which is a contradiction.

In the case that $\varepsilon<\left|\gamma-\gamma^{\prime}\right| /\left|\gamma+\gamma^{\prime}\right|$ suppose the $\varepsilon$ sheath about $\Gamma\left(f, \gamma^{\prime}\right)$ intersects $\Gamma(f, \gamma)$ arbitrarily close to 1 . Then, we find a net $r_{\alpha} e^{i \theta_{\alpha}} \in \Gamma(f, \gamma)$ tending to a nontrivial homomorphism $h$ and corresponding points $r_{\alpha}^{\prime} e^{i \theta_{\alpha^{\prime}}} \in \Gamma\left(f, \gamma^{\prime}\right)$ tending, say, to $h^{\prime}$ with $\chi\left(r_{\alpha} e^{i \theta_{\alpha}}, r_{\alpha}^{\prime} e^{i \theta_{\alpha^{\prime}}}\right)<\varepsilon$. Using the first part of the present theorem and the lower semicontinuity of $\chi$,

$$
\left|\left(\gamma-\gamma^{\prime}\right) /\left(\gamma+\gamma^{\prime}\right)\right| \leqq \chi\left(h, h^{\prime}\right) \leqq \varepsilon<\left|\left(\gamma-\gamma^{\prime}\right) /\left(\gamma+\gamma^{\prime}\right)\right| ;
$$

again a contradiction.

Although we intended this note to contain only intrinsic results concerning approaches along convex curves we cannot resist the following application which generalizes the classical result that an $H^{\infty}$ function which tends to zero along the radius also tends to zero along each Stolz ray.

THEOREM 7. Let $f \in \mathscr{K}$ and let $g$ be a bounded analytic function on $D$. If $g$ tends to 0 asymptotically along one curve $\Gamma(f, \gamma), 0<\gamma<\infty$, then it tends to zero along all such curves.

Proof. If $P$ is any part in $A(f)$, then $g=0$ on $A(f, \gamma) \cap P$. The latter set being the image of an oricycle under the Wermer map, $\tau$, from $D$ onto $P$, the analytic function $g \circ \tau$ vanishes identically on $D$. That is, $g=0$ on $P$ and therefore on $A(f)$.

\section{REFERENCES}

1. T. K. Boehme and Max L. Weiss, One-sided boundary behavior for certain harmonic functions, Proc. Amer. Math. Soc. 27 (1971), 280-288. MR 44 \#5491.

2. K. Hoffman, Bounded analytic functions and Gleason parts, Ann. of Math. (2) 86 (1967), 74-111. MR 35 \#5945.

3. M. Rosenfeld and Max L. Weiss, Bounded analytic functions tending radially to zero, Proc. London Math. Soc. (3) 18 (1968), 714-726. MR 38 \#1269.

4. M. L. Weiss, Note on a theorem of Beurling, Proc. Amer. Math. Soc. 19 (1968), 793-795. MR 37 \#3006.

5. Dennis H. Wortman, Interpolating sequences in convex regions of the open unit disc, Doctoral Dissertation, M.I.T , Cambridge, Mass., 1972.

Department of Mathematics, California State University, Northridge, CALIFORNia 91324

Department of Mathematics, University of California, Santa Barbara, CALIFORNIA 93106 